\title{
ENHANCEMENT OF AODV ROUTING PROTOCOL IN MASNETS
}

\author{
M. N. Jambli*, W. B. Wan Mohd Shuhaimi *, H. Lenando*, J. Abdullah*, S. Mohamad Suhaili ${ }^{\dagger}$ \\ *Faculty of Computer Science \& Information Technology, Universiti Malaysia Sarawak, 94300 Sarawak, Malaysia \\ jmnazim@fit.unimas.my, wan.balkis@gmail.com, cool@fit.unimas.my, ajohari@fit.unimas.my \\ $\dagger$ Centre for Pre-University Studies, Universiti Malaysia Sarawak, 94300 Sarawak, Malaysia \\ mssinarwati@preuni.unimas.my
}

\begin{abstract}
The rapid development of wireless communication technologies and portable mobile devices such as laptops, PDAs, smart phones and wireless sensors brings the best out of mobile computing particularly mobile ad-hoc and sensor networks. Nowadays, most of researchers working on Wireless Sensor Networks (WSNs) focus on Mobile Ad-hoc and Sensor Networks (MASNETs) due to their wide range of potential applications ranging from underwater monitoring to search and rescue mobile robotics applications. In this research work, through extensive simulation, we have evaluated the capability of Ad-hoc On Demand Distance Vector (AODV) routing protocol on how far it can react to different mobility duration of mobile nodes in MASNETs. The performance of AODV is investigated in terms of the average percentage of packet loss and energy consumption. The initial performance study demonstrates that the performance of AODV is signicantly decreased in mobile environment due to the frequent topology change in MASNETs. Therefore, in order to enhance the performance of AODV in MASNETs, the new routing algorithm based on the estimated distance is proposed to replace the hop count for the selection of next node during the packet transmission. The estimated distance is computed based on the Received Signal Strength Indicator (RSSI) values that are collected during the communication between the sensor nodes. The performance of the proposed solution is also further evaluated and compared with the existing AODV routing protocol in term of their energy consumption and percentage of packet loss. Our study demonstrates that the proposed solution outperform the existing AODV in MASNETs.
\end{abstract}

Index Terms-MASNETs, Ad-hoc On-Demand Distance Vector, Distance Estimation.

\section{INTRODUCTION}

Recently, extensive research has been conducted on Mobile Ad-Hoc Sensor Networks (MASNETs) due to their wide range of potential applications ranging from environmental monitoring, animal monitoring to critical military surveillance. These applications can be executed either in static or mobile environment. In static networks, the change of sensor nodes topology is normally caused by node failure which is due to energy depletion. However, in MASNETs, the main reason of the topology change is caused by the node movement. Since the sensor nodes are limited in power supply and have low radio frequency coverage, they are easily losing their connection with neighbours and have difficulties to transmit their packets towards sink node[1]. The reconnection process from one node to another node consumes more energy that related to control packets. Random nodes movement in MASNETs application shows degradation in network performance due to high speed of mobile nodes.

Power consumption is one of the critical issue for routing in mobile ad hoc sensor networks(MASNETs)[2]. MASNETs is a network which contains mobile nodes that topology constantly changing. The main concerns is on how to select the next hop node that support energy conservation as it will certainly provide reliable and good path for data forwarding since mobility may cause radio links to break frequently. Most literature used shortest path (measured in terms of links or number of hops that a path goes through) as the major routing metric. Due to the vast dynamic nature of wireless channel, traditional metrics used such as hop count generally hard to provide a highly reliable path estimation in Wireless Sensor Networks (WSNs) and most probably consumed more energy in communication. This research is focus on analysing how far the proposed energy-savvy routing algorithm for MASNETs based on estimated distance improve the performance of AODV in mobile environment in terms of energy consumption and percentage of packet loss.

In this paper, through extensive simulation we evaluated the capability of AODV on how far it can react to network topology change in MASNETs. We investigated the performance metrics namely energy consumption and percentage of packet loss of mobile nodes with various simulation time. The rest of the paper is organized as follows: Section II includes the recent related works of current routing algorithm. The AODV routing protocol description is summarized in section III. The simulation environment and set-up are described in Section IV. We present the simulation results in section V. Section VI concludes the paper and outlines the future work.

\section{RELATED WORK}

In recent past, several works have been done on MASNETs along with the evolvement of wireless sensor network [3]. Many works have done particularly in mobile environment networks involving distance or location estimation using various approaches in MASNETs. Estimated distance is used to select the nearest next hop node in mobile environment as the nodes movement are effecting the process of selecting the next hop node for forwarding message from source to destination. Due 